\title{
Microbiolização na qualidade de sementes e crescimento inicial de plantas de Vigna unguiculata L. Walp
}

\author{
Mylenna Nádja Ferreira de Sáa*, Jéssica de Souza Limab, Fábio Nascimento de Jesus ${ }^{b}$, Jane \\ Oliveira Perez \\ a Instituto Federal de Educação, Ciência e Tecnologia do Sertão Pernambucano, Petrolina, 56302-970, Pernambuco, \\ Brasil.mylennadjafs@gmail.com* \\ b Instituto Federal do Sertão Pernambucano Petrolina Zona Rural, Petrolina, 56302-970, Pernambuco, Brasil.
}

Recebido: 6 fevereiro 2019 / Aceito: 01 maio 2019 / Publicado online: 30 setembro 2019

\begin{abstract}
Resumo
Vigna unguiculata L. Walp (feijão-caupi) tem grande importância como fonte de alimento e como gerador de emprego e renda para a população rural brasileira. $\mathrm{O}$ tratamento de sementes por meio da microbiolização pode beneficiar a germinação de sementes, emergência e desenvolvimento das plântulas e produção de grãos. Objetivou-se com esta pesquisa avaliar o efeito da microbiolização na germinação e sanidade de sementes, assim como o crescimento inicial de plantas de Vigna unguiculata $\mathrm{L}$. Walp cv. BRS Pujante. Os organismos utilizados na microbiolização das sementes foram Bacillus subtilis (LCB30), Bacillus subtilis (LCB45), Bacillus sp. (BMH), Bacillus sp. (INV) e Trichoderma sp. Avaliou-se o percentual de germinação, o crescimento da radícula e a incidência de fungos nas sementes microbiolizadas. Em viveiro, foram utilizados os mesmos microrganismos para microbiolização em um delineamento experimental inteiramente casualizado, com 4 repetições para teste de germinação, e 10 repetições para teste em casa de vegetação. Após 51 dias de crescimento das plantas, foram analisadas a massa fresca da parte aérea (MFPA) e da raiz (MFR), massa seca da parte aérea (MSPA) e da raiz (MSR) e a contagem de nódulos (NN). Todos os tratamentos proporcionaram o incremento na germinação de sementes de $V$. unguiculata BRS Pujante, exceto INV, com apenas $81 \%$ das sementes germinadas. Todas as sementes microbiolizadas reduziram a incidência de fungos. A microbiolização das sementes com os diferentes microrganismos não apresentou eficiência na nodulação das plantas. $\mathrm{O}$ isolado LCB 30 promoveu aumento significativo na MSPA e MSR de plantas de V. unguiculata.
\end{abstract}

Palavras-chave: Bacillus subtilis, germinação, feijão-caupi.

\section{Microbiolization in seed quality and initial growth of Vigna unguiculata L. Walp plants}

\begin{abstract}
Vigna unguiculata L Walp (cowpea bean) has great importance as a source of food and as a generator of employment and income for the brazilian rural population. Seed treatment through microbiolization, is beneficial in seed germination, seedling emergence and development, and grain production. The objective of this work was to evaluate the effect of microbiolization on seed germination and sanity, as well as the initial growth of Vigna unguiculata BRS Pujante plants. The organisms used in the microbiolization of the seeds were Bacillus subtilis (LCB30), Bacillus subtilis (LCB45), Bacillus sp. (BMH), Bacillus sp. (INV) and Trichoderma sp. The percentage of germination, the growth of the radicle and the incidence of fungi in the microbiolized seeds were evaluated. The same microorganisms were used for microbiolization in the nursery test. Fresh mass of the aerial part (MFPA), root (MFR), shoot dry mass (MSPA) and root (MSR), and nodule count (NN) were analyzed after 51 days of plant growth. For the analyzes, the DIC was used, with 4 replicates for germination test, and 10 replicates for greenhouse testing. All treatments provided an increase in germination of $V$. unguiculata BRS Pujante seeds, except for INV, with only $81 \%$ of germinated seeds. However, in the sanitary analysis, all microbiolized seeds reduced the incidence of fungi. The microbiolization of the seeds with the different microorganisms was not efficient in the nodulation of the plants. The LCB 30 isolate promoted a significant increase in MSPA and MSR of $V$. unguiculata plants.
\end{abstract}

Keywords: Bacillus subtilis, germination, cowpea bean.

\section{Introdução}

A $V$. unguiculata (feijão-caupi) é uma das leguminosas mais consumidas e cultivadas no Brasil, com destaque para as regiões Norte e Nordeste. É amplamente produzida por pequenos produtores, constituindo um dos principais componentes da dieta alimentar, especialmente na zona rural 
(Silva, Santos, \& Santos, 2018). A produção brasileira para o ano agrícola 2017/2018 foi de 848,3 mil toneladas de grãos (Companhia Nacional de Abastecimento [CONAB], 2018), configurando o Brasil como o terceiro produtor mundial.

Apesar da importância da cultura, no Brasil, sua produtividade média ainda é baixa, em função do baixo nível tecnológico empregado no cultivo (Oliveira, Schuch, Bruno, $\&$ Peske, 2015). Neste sentido, a utilização de sementes com potencial fisiológico elevado é fundamental para o estabelecimento de qualquer lavoura, pois o uso de sementes de baixo potencial de germinação e vigor reduzido originam lavouras com baixa população de plantas e consequentemente baixa produtividade (Krzyzanowski, Gilioli, \& Miranda, 1993).

A aplicação de microrganismos no tratamento de sementes tem sido utilizada visando o aumento da produtividade, devido proteger as plantas contra fitopatógenos e promover o crescimento de plantas. De modo geral, esses mecanismos estão relacionados ao desenvolvimento das plantas, incluindo os efeitos benéficos na germinação de sementes, emergência e desenvolvimento das plântulas e produção de grãos e frutos (Harman, 2000). Outros ganhos secundários têm sido observados no solo, na ação sobre nutrientes indisponíveis, que passam a estar facilmente disponíveis e, também melhorias na qualidade estrutural (Junges, 2012).

A técnica de microbiolização tem sido utilizada com resultados satisfatórios e tem se apresentado como uma estratégia viável para a redução do uso exclusivo de produtos sintéticos, proporcionando benefícios econômicos e ambientais (Junges et al., 2014). Entre os microrganismos mais utilizados, em termos mundiais, destacam-se Agrobacterium radiobacter, Bacillus subtilis, Pseudomonas fluorescens, Trichoderma harzianum e Gliocladium roseum (Lazzaretti \& Bettiol, 1997).

O tratamento de sementes com Trichoderma spp. apresenta-se como uma alternativa para o controle de doenças em plantas, estimular o enraizamento e o crescimento vegetal, promover a germinação de sementes e, ainda, acelerar o estabelecimento de mudas no campo após o transplantio (Luz, 1996). Bactérias do gênero Bacillus possuem mecanismos diversos sobre as plantas tais como: produção de fitohormônios e sideróforos, indução de resistência, antibiose e parasitismo (Ramamoorthy, Viswanathan, Raguchander, Prakasam, \& Samiappan, 2001; Tarnawski, Hamelin, Jossi, Aragno, \& Fromin, 2006).

Dessa forma, objetivou-se com esta pesquisa avaliar o efeito da microbiolização na germinação e sanidade de sementes, assim como o crescimento inicial de plantas de V. unguiculata BRS Pujante.

\section{Material e Métodos}

A pesquisa foi desenvolvida no Instituto Federal do Sertão Pernambucano, campus Petrolina Zona Rural (IF Sertão-PE).

As sementes de $V$. unguiculata cv. BRS Pujante foram adquiridas na Embrapa Semiárido, Petrolina-PE. Na mesma instituição, laboratório de controle biológico da Embrapa Semiárido foram adquiridos os organismos utilizados na microbiolização das sementes (Tabela 1).

Os isolados bacterianos foram multiplicados em placas de 112
Petri contendo meio de cultura ágar nutriente (AN) e mantidos em câmara BOD (Demanda Bioquímica de Oxigênio) para crescimento por 48 horas a $28^{\circ} \mathrm{C}$. Para obtenção das suspensões bacterianas foi adicionada solução salina estéril $(0,85 \%)$ nas placas e realizada a raspagem com alça de Drigalski e coletada em tubos de Eppendorf® $(2000 \mu \mathrm{L})$. Em seguida, a suspensão final de cada bactéria foi ajustada de acordo com a leitura realizada em espectrofotômetro da marca BEL Photonics, modelo V-M5 comprimento de onda de 600nm e absorbância de 0,5).

Tabela 1. Microrganismos utilizados para microbiolização de sementes de Vigna unguiculata cv. BRS Pujante.

\begin{tabular}{cl}
\hline Tratamento & \multicolumn{1}{c}{ Microrganismo } \\
\hline 1 & Bacillus sp. (BMH) \\
2 & Bacillus subtilis (LCB 30) \\
3 & Bacillus subtilis (LCB 45) \\
4 & Bacillus sp. (INV) \\
5 & Trichoderma sp. \\
6 & Testemunha - Água destilada \\
\hline
\end{tabular}

A suspensão de Trichoderma sp. foi obtida pela adição de $20 \mathrm{~mL}$ de água destilada esterilizada (ADE) e 1 gota de Tween ${ }^{\circledR}$ sob colônias do fungo crescidas em placas de Petri contendo meio de cultura batata dextrose e ágar (BDA) por 10 dias a $28^{\circ} \mathrm{C}$. A concentração final foi ajustada para $10^{7}$ conídios $\mathrm{mL}^{-1}$ por meio de contagens em câmara de Neubauer.

Antes do início dos testes, as sementes foram desinfestadas superficialmente por meio da imersão em solução de hipoclorito de sódio $(\mathrm{NaOCl} 1 \%)$ por $1 \mathrm{~min}$. e, logo após, em álcool a $70 \%$ (1 min.), seguida de três lavagens sucessivas com água destilada esterilizada e secagem natural em papel absorvente.

Para a microbiolização foi adotada a metodologia adaptada por Bezerra et al. (2013), onde 50 sementes em cada replicata foram imersas em cada uma das suspensões $(70 \mathrm{~mL})$ durante 60 minutos. Para a testemunha, as sementes foram imersas somente em $70 \mathrm{~mL}$ de ADE. O delineamento estatístico foi inteiramente casualizado com quatro repetições.

As sementes microbiolizadas foram submetidas ao teste de germinação em papel tipo rolo germitest, umedecidos com água destilada estéril na proporção de 2,5 vezes ao peso do papel seco e colocadas para germinar em BOD a $25^{\circ} \mathrm{C}$, conforme as Regras para Análise de Sementes (Brasil, 2009).

No quarto e sétimo dia após a instalação do teste, foi avaliado o número de sementes germinadas para calcular a porcentagem de germinação (BRASIL, 2009). A partir do teste de germinação, também foi realizado o teste de sanidade das sementes, onde determinou-se a incidência (\%) de sementes infectadas por fungos patogênicos, e estes foram identificados a nível de gênero sob lupa ou microscópico óptico. Ao final do período de sete dias, dez plântulas de cada repetição foram escolhidas ao acaso para avaliar o comprimento da radícula com auxílio de um paquímetro digital, sendo um total de 240 plântulas avaliadas. 
No ensaio em casa de vegetação, foram utilizados 10 sacos de plantio por tratamento (com capacidade para 3 litros) contendo solo agrícola, sendo cada saco uma repetição, e 3 sementes microbiolizadas por saco. As sementes utilizadas no experimento na casa de vegetação foram diferentes das sementes utilizadas no teste de germinação, pois os experimentos foram realizados em períodos diferentes.

O preparo da suspensão de Trichoderma sp. $\left(10^{7}\right.$ conídios $\mathrm{mL}^{-1}$ ) foi o mesmo realizado para o teste de germinação de sementes. Entretanto, para obtenção das suspensões das bactérias, os isolados cultivados em meio Agar Nutriente (48 horas) foram colocados com auxílio de uma alça inoculadora em Erlenmeyers com $125 \mathrm{~mL}$ de caldo nutriente e mantidos por 24 horas em mesa agitadora da marca TECNAL, modelo TE-145, para o crescimento. Logo após, as suspensões foram padronizadas em espectrofotômetro $(\mathrm{A}=600 \mathrm{~nm})$ para 0,5 .

Para o plantio foram utilizadas 3 sementes por saco e $3 \mathrm{~mL}$ da suspensão em cada repetição, por respectivo tratamento. Após 20 dias após a semeadura (DAS), foi realizado o desbaste, permanecendo uma planta por saco. E 30 dias após a semeadura foi realizado a reaplicação das suspensões.

Após 51 dias da semeadura as plantas foram coletadas e as raízes e parte aérea foram separadas, sendo as raízes lavadas em água corrente para separação do solo. Foi realizada a contagem de nódulos presentes em todas as raízes. Para obtenção da massa seca, as partes vegetais foram acondicionados em sacos de papel e secos em estufa de circulação forçada a $60^{\circ} \mathrm{C}$, por 24 horas. Em balança analítica de precisão, foi aferida a massa seca da parte aérea (MSPA) e das raízes (MSR) e os resultados expressos em gramas. Para as análises foi utilizado o delineamento inteiramente casualizado, com dez repetições.

Os dados obtidos foram submetidos à análise de variância (ANOVA), e tiveram as médias comparadas pelo teste de Scott-Knott, em nível de 5\% probabilidade (Ferreira, 2000).

\section{Resultados e Discussão}

Os isolados bacterianos Bacillus sp. (BMH), B. subtilis (LCB 30), B. subtilis (LCB 45) e Trichoderma sp. promoveram aumento a germinação das sementes quando comparada a testemunha (Tabela 2). No entanto, as sementes germinadas microbiolizadas com Bacillus sp. (INV) apresentou percentual de germinação inferior aos demais tratamentos e à testemunha.

Estudos desenvolvidos por Rocha, Sakai, Souza, Junior e Santos (2017) mostram resultados semelhantes onde isolados de Trichoderma asperellum e B. subtilis demonstraram o seu potencial na elevação da germinação e velocidade de emergência de sementes de $V$. unguiculata Sempre Verde e BRS Verde. O mesmo foi reportado por Marques, Santos, Silva, Martins e Mello (2014), para sementes de Vigna unguiculata $\mathrm{cv}$. BRS Estilo, quando submetidas ao tratamento com isolados de Trichoderma spp., onde apresentaram maior percentagem e precocidade germinativa em relação à testemunha.

Os efeitos de bactérias do gênero Bacillus spp. são diversos, incluindo potencial ação na germinação e emergência de plântulas, auxiliam na fixação de nitrogênio, produzem hormônios importantes para o crescimento vegetal
(Cerqueira, Morais, Miranda, \& Mello, 2015). Em alguns trabalhos, observou-se que o aumento no percentual de germinação, índice de velocidade de germinação e crescimento de plantas expostas a essa bactéria, tem sido relacionado a produção de giberelinas, auxinas e ácidos láticos succínio (Holl, Chanway, Turkington, \& Radley, 1988; Yoshikawa, Hirai, Wakabayashi, Sugizaki, \& Iwamura, 1993).

As radículas com maiores comprimentos (CR), foram verificadas nas sementes tratadas com B. subtilis (LCB 45) e B. subtilis (LCB 30);(Tabela 2). No entanto, notou-se menores valores para comprimento nas sementes tratadas com Bacillus sp. (BMH) e Bacillus sp. (INV), não diferindo da testemunha. Conforme Freitas et al., (2005) e Nakagawa (1999) a avaliação do comprimento de radícula tem como princípio de que lotes com sementes que emitem radícula e hipocótilo em menor tempo são mais vigorosos e aptos as condições de estresse ambiental, garantindo maiores sobrevivência no campo. Os resultados obtidos corroboram com os de Oliveira, Silva, Marciano, Proença e Sá (2016), onde sementes de $V$. unguiculata cv. BRS Estilo de alto vigor submetidas a diferentes dosagens de um produto a base de B. subtilis apresentaram aumento no comprimento de plântula e de raiz primária. Essa influência direta no crescimento vegetal deve ser associada ao estímulo dos microrganismos benéficos na produção de fitohormônios como, por exemplo, a auxina.

Tabela 2. Germinação de sementes (\%), comprimento de radícula $(\mathrm{cm})$ e incidência de fungos $(\%)$ em sementes de Vigna unguiculata cv. BRS Pujante microbiolizadas com diferentes microrganismos.

\begin{tabular}{lccc}
\hline \multicolumn{1}{c}{ Tratamento } & Germinação & Radícula & Incidência \\
\hline Bacillus sp. BMH & $98,00 \pm 2,8^{\mathrm{a}}$ & $1,05 \pm 0,13^{\mathrm{d}}$ & $8,25 \pm 4,57^{\mathrm{a}}$ \\
B. subtilis LCB 30 & $100,00 \pm 0,0^{\mathrm{a}}$ & $4,36 \pm 0,23^{\mathrm{b}}$ & $6,75 \pm 2,87^{\mathrm{a}}$ \\
B. subtilis LCB 45 & $99,50 \pm 1,0^{\mathrm{a}}$ & $5,59 \pm 0,32^{\mathrm{a}}$ & $11,00 \pm 2,58^{\mathrm{a}}$ \\
Bacillus sp. INV & $81,00 \pm 4,8^{\mathrm{c}}$ & $1,61 \pm 0,28^{\mathrm{d}}$ & $16,00 \pm 3,56^{\mathrm{b}}$ \\
Trichoderma sp. & $99,00 \pm 2,0^{\mathrm{a}}$ & $2,08 \pm 0,52^{\mathrm{c}}$ & $18,25 \pm 7,59^{\mathrm{b}}$ \\
Testemunha & $93,00 \pm 3,5^{\mathrm{b}}$ & $1,32 \pm 0,18^{\mathrm{d}}$ & $40,75 \pm 7,41^{\mathrm{c}}$ \\
\hline Médies
\end{tabular}

Médias seguidas de mesma letra, na coluna, não diferem entre si pelo teste de Scott-Knott à 5\% de probabilidade.

$\mathrm{Na}$ análise da sanidade de sementes de $V$. unguiculata microbilizadas, foram identificados os seguintes fungos, a nível de gênero: Aspergillus spp., Penicillium spp., Fusarium spp. e Rhizopus spp. Observou-se que a maior incidência de fungos patogênicos ocorreu no tratamento testemunha (ausência de microrganismo) com percentual de incidência de 40,75\% (Tabela 2). Os menores percentuais de incidência foram observados nas sementes microbiolizadas com Bacillus sp. (BMH), B. subtilis (LCB 30) e B. subtilis (LCB 45) sendo, respectivamente $8,25 \% ; 6,75 \%$ e $11,00 \%$.

Conforme Machado (2000), no manejo integrado de doenças o tratamento sanitário de sementes com agentes biocontroladores tem sido uma prática recomendada por controlar doenças na fase que antecede a implantação da cultura, possibilitando redução do uso de defensivos químicos. Ethur, Rocha, Milanesi, Marlove e Blume (2006) sugerem que caso essas sementes fossem semeadas, as estruturas desses microrganismos benéficos poderiam se estabelecer no solo, 
tornando-o supressivo a diversos patógenos.

Os resultados sugerem que os organismos utilizados na microbiolização de sementes de $V$. unguiculata possivelmente apresentaram, em algum nível, ação biocontroladora de patógenos que causam danos em sementes. Bactérias antagônicas, como B. subtilis, de modo geral agem significativamente por antibiose e, ocasionalmente, por parasitismo e competição, e seus isolados produzem uma grande variedade de metabólitos antifúngicos, entre os quais se encontram lipopeptídeos das famílias da surfactina, iturina e fengicina (Lanna Filho, Ferro, \& Pinho, 2010).

A capacidade do antagonista Trichoderma spp. de controlar fitopatógenos presentes no solo, tem sido associado, principalmente, aos mecanismos de micoparasitismo, antibiose e competição (Harman, 2006).

Para a variável massa fresca da parte aérea (MFPA), observou-se que não houve diferença entre os tratamentos (Tabela 3). As massas verificadas variaram de 2,44 a 3,95 g planta $^{-1}$, entretanto, essa variabilidade não foi estatisticamente diferente. Para a matéria fresca de raiz (MFR) os tratamentos contendo Trichoderma sp. (2,40 $\left.\mathrm{g}_{\text {planta }}{ }^{-1}\right)$ e Bacillus sp. (INV) (1,96 $\left.\mathrm{g} \mathrm{planta}^{-1}\right)$ reportaram valores estatisticamente superiores aos demais tratamentos. Assim como os resultados obtidos por Machado, Tavares, Lopes e Silva (2015) onde diferentes isolados de Trichoderma viride e Trichoderma harzianum incrementaram as matérias frescas de parte aérea e de raiz em mudas de cambará (Gochnatia polymorpha).

Tabela 3. Massa fresca de parte aérea (MFPA) e massa fresca de raiz (MFR) de plantas de Vigna unguiculata cv. BRS Pujante submetidas a microbiolização durante a semeadura e durante crescimento vegetativo.

\begin{tabular}{lcc}
\hline \multicolumn{1}{c}{ Tratamento } & MFPA $(\mathrm{g})$ & MFR $(\mathrm{g})$ \\
\hline Bacillus sp. BMH & $3,28 \pm 1,08^{\mathrm{a}}$ & $0,98 \pm 0,26^{\mathrm{b}}$ \\
B. subtilis LCB 30 & $3,95 \pm 1,60^{\mathrm{a}}$ & $0,94 \pm 0,49^{\mathrm{b}}$ \\
B. subtilis LCB 45 & $2,89 \pm 0,66^{\mathrm{a}}$ & $1,39 \pm 0,74^{\mathrm{b}}$ \\
Bacillus sp. INV & $2,94 \pm 1,55^{\mathrm{a}}$ & $1,96 \pm 0,63^{\mathrm{a}}$ \\
Trichoderma sp. & $3,17 \pm 0,92^{\mathrm{a}}$ & $2,40 \pm 1,07^{\mathrm{a}}$ \\
Testemunha & $2,44 \pm 0,64^{\mathrm{a}}$ & $1,52 \pm 0,71^{\mathrm{b}}$ \\
\hline
\end{tabular}

Médias seguidas de mesma letra, na coluna, não diferem entre si pelo teste de Scott-Knott à 5\% de probabilidade.

Para a massa seca da parte aérea (MSPA), o tratamento $B$. subtilis (LCB 30) apresentou maior massa seca (2,88 $\mathrm{g} \mathrm{planta}^{-}$ $\left.{ }^{1}\right)$ quando comparado aos demais tratamentos (Tabela 4). Resultado semelhante foi observado por Chagas et al., (2017) que ao avaliaram o efeito do uso B. subtilis (UFT B10) como promotor de crescimento vegetal na cultura de $V$. unguiculata observaram que aplicação isolada da bactéria promoveu incremento da MSPA das plantas, com 1,07 g planta ${ }^{-1}$. Conforme Lazzareti (2005), constatou que o uso de B. subtilis é uma estratégia promissora para promover crescimento de plantas de feijoeiro.

A aplicação dos tratamentos Trichoderma sp., B. subtilis (LCB 30) e Bacillus sp. (INV) nas sementes de V. unguiculata promoveram o aumento significativo da massa seca da raiz (MSR), obtendo-se os valores 0,52; 0,42 e 0,40 g planta $^{-1}$ respectivamente (Tabela 4 ).

Oliveira et al. (2016) verificou que diferentes doses de um 114 produto a base de B. subtilis associada a sementes de alto vigor, promoveram maior acúmulo de MSPA, com 0,2714 g planta $^{-1}$, e MSR, com 0,1027 $\mathrm{g} \mathrm{planta}^{-1}$, de plantas de feijoeiro cv. BRS Estilo.

Os resultados obtidos em ambos os trabalhos, este e o de Oliveira et al., podem estar relacionados ao fato de que $B$. subtilis induzir na planta a síntese de fitohormônios, como ácido indolacético, ácido abscísico, giberelinas e citocininas que favorecem o crescimento das raízes e o aumento no número dos pelos radiculares (Araújo, Carneiro, Bezerra, \& Araújo, 2009). Outros estudos têm sido realizados com diferentes linhagens de Trichoderma spp. demonstrando promoção de crescimento de plantas pelo aumento na disponibilidade de nutrientes e produção de hormônios e crescimento (Kapri e Tewari, 2010; Chagas, Chagas Junior, Carvalho, Miller, Colonia 2015).

Tabela 4. Massa seca de parte aérea (MSPA), de raiz (MSR) e percentual de nodulação (NN) de plantas de Vigna unguiculata cv. BRS Pujante submetidas a microbiolização durante a semeadura e durante crescimento vegetativo.

\begin{tabular}{lccc}
\hline \multicolumn{1}{c}{ Tratamento } & MSPA $(\mathrm{g})$ & MSR $(\mathrm{g})$ & $\mathrm{NN}(\%)$ \\
\hline Bacillus sp. BMH & $1,35 \pm 0,54^{\mathrm{b}}$ & $0,20 \pm 0,06^{\mathrm{b}}$ & 50,00 \\
B. subtilis LCB 30 & $2,88 \pm 1,39^{\mathrm{a}}$ & $0,42 \pm 0,19^{\mathrm{a}}$ & 50,00 \\
B. subtilis LCB 45 & $1,76 \pm 0,71^{\mathrm{b}}$ & $0,29 \pm 0,17^{\mathrm{b}}$ & 50,00 \\
Bacillus sp. INV & $1,43 \pm 0,77^{\mathrm{b}}$ & $0,40 \pm 0,17^{\mathrm{a}}$ & 50,00 \\
Trichoderma sp. & $1,69 \pm 0,47^{\mathrm{b}}$ & $0,52 \pm 0,24^{\mathrm{a}}$ & 100,00 \\
Testemunha & $1,09 \pm 0,46^{\mathrm{b}}$ & $0,27 \pm 0,09^{\mathrm{b}}$ & 30,00
\end{tabular}

Médias seguidas de mesma letra, na coluna, não diferem entre si pelo teste de Scott-Knott à $5 \%$ de probabilidade.

Em todos os tratamentos observou-se a ocorrência de nódulos, mas algumas plantas microbiolizadas e não microbiolizadas de $V$. unguiculata avaliadas não apresentaram nodulação (NN), exceto aquelas tratadas com Trichoderma sp., tratamento que obteve maior percentual de nodulação (Tabela 4). Resultados contrários foram obtidos por Araújo et al., (2009) demonstraram aumento na nodulação de $V$. unguiculata quando coinoculado com $B$. subtilis e Bradyrhizobium, atribuindo-se influência positiva do Bacillus pela competição com o rizóbio, aumentando os sítios de infecção e a ação inibitório do crescimento de fungos nas raízes.

\section{Conclusões}

A microbiolização com Bacillus sp. (BMH), B. subtilis (LCB 30), B. subtilis (LCB 45) e Trichoderma sp. promoveu discreto aumento da germinação nas sementes de Vigna unguiculata cv. BRS Pujante, além disso, para as sementes expostas ao B. subtilis (LCB 45) houve maior comprimento da radícula e redução da incidência de fungos nas sementes. Entretanto, a microbiolização não foi eficiente para aumentar nodulação. O isolado B. subtilis (LCB 30) se destacou por promover aumento na massa seca da parte aérea e da raiz de Vigna unguiculata.

\section{Referências}

Araújo, A. S. F., Carneiro, R. F. V., Bezerra, A. A. C., \& Araújo, F. F. (2009). 
Coinoculação rizóbio e Bacillus subtilis em feijão-caupi e leucena: efeito sobre a nodulação, a fixação de N2 e o crescimento das plantas. Ciência Rural, 40(1), 182-185. doi:10.1590/S0103-84782009005000249

Bezerra, G. A., Macedo, D. A., Nascimento, I. O., Sousa, T. P., Costa, N. B., \& Sousa, L. F. R. A. (2013). Uso de Bacillus spp. no controle de fitopatógenos em sementes de soja variedade BRS Valiosa RR. Revista Agroecossistemas, 5(1), 68-73. doi:10.18542/ragros.v5i1.1414

Brasil. (2009). Ministério da Agricultura, Pecuária e Abastecimento. Regras para Análise de Sementes. Ministério da Agricultura, Pecuária e Abastecimento. Secretaria de Defesa Agropecuária. Brasília, DF: Mapa/ACS, pp. 398.

Cerqueira, W. F., Morais, J. S., Miranda, J. S., \& Mello, I. K. S. (2015). Influência de bactérias do gênero Bacillus sobre o crescimento de feijãocomum (Phaseolus vulgaris L.). Enciclopédia Biosfera, 11(20), pp. 82.

Chagas, L. F. B., Chagas Junior, A. F., Carvalho, M. R.., Miller, L. O., \& Colonia, B. S. O. (2015). Evaluation of the phosphate solubilization potential of Trichoderma strains (Trichoplus JCO) and effects on rice biomass. Journal of Soil Science and Plant Nutrition, 15(3), 794-804. doi:10.4067/S0718-95162015005000054

Chagas, L. F. B., Martins, A. L. L., Carvalho Filho, M. R. de, Miller, L. O., Oliveira, J. C., \& Chagas Junior, A. F. (2017). Bacillus subtilis e Trichoderma sp. no incremento da biomassa em plantas de soja, feijãocaupi, milho e arroz. Agri-environmental sciences, 3(2), 10-18.

Companhia Nacional de Abastecimento. (2018). Acompanhamento da safra brasileira de grãos: safra 2016/17 e 2017/18 décimo primeiro levantamento. Brasília: Conab.

Ethur, L. Z., Rocha, E. K., Milanesi, P., Marlove, F. B. M, \& Blume, E. (2006). Sanidade de sementes e emergência de plântulas de nabo forrageiro, aveia preta e centeio submetidas a tratamentos com bioprotetor e fungicida. Ciência e Natura, 28(2), 17-27. doi:10.5902/2179460X9700

Ferreira, D. F. (2000). Análise estatística por meio do SISVAR (Sistema para Análise de Variância) para Windows versão 4.0. In: Reunião Anual da Região Brasileira da Sociedade Internacional de Biometria, 255- 258.

Freitas, T. A. S., Barroso, D. G., Carneiro, J. G. A., Penchel Filho, R. M., Lamônica, K. R., \& Ferreira, D. A. (2005). Desempenho radicular de mudas de eucalipto produzidas em diferentes recipientes e substratos. Revista Árvore, 29(6), 853-861. doi:10.1590/S010067622005000600003

Harman, G. E. (2000). Myth and dogmas of biocontrol changes in perceptions derived from research on Trichoderma harzianum T-22. Plant Diasease, 84(4), 377-393. doi:10.1094/PDIS.2000.84.4.377

Harman, G. E. (2006). Overview of mechanisms and uses of Trichoderma spp. Phytopathology, 96, 190-194. doi:10.1094/PHYTO-96-0190

Holl, F. B., Chanway, C. P., Turkington, R., \& Radley, R. A. (1988). Response of crested wheatgrass (Agropyron cristatum L.), perennial ryegrass (Lolium perenne) and white clover (Trifolium repens L.) to inoculation with Bacillus polymyxa. Soil Biology and Biochemistry, 20, 19-24. doi:10.1016/0038-0717(88)90121-6

Junges, E., Bastos, B. O., Toebe, M., Muller, J., Pedroso, D. C., \& Muniz, M. F. B. (2014). Restrição hídrica e peliculização na microbiolização de sementes de milho com Trichoderma spp. Comunicata Scientiae, 5(1), $18-25$.

Junges, E. (2012). Técnicas de microbiolização de sementes de milho, feijão, nabo forrageiro e aveia preta. (Dissertação de Mestrado). Universidade Federal de Santa Maria, Santa Maria, Rio Grande do Sul.

Kapri, A., \& Tewari, L. (2010). Phosphate solubilization potential and phosphatase activity of rhizospheric Trichoderma spp. Brazilian Journal of Microbiology 41(3),787-795. doi:10.1590/S151783822010005000001

Krzyzanowski, F. C., Gilioli, J. L., \& Miranda, L. C. (1993). Produção de sementes nos cerrados. In: Arantes, N.E. \& Souza, P.I.M. Cultura da soja nos cerrados. Piracicaba: Potafos, pp.465-522.

Lanna Filho, R., Ferro, H. M., \& Pinho, R. S. C. (2010). Controle biológico mediado por Bacillus subtilis. Revista Trópica: Ciências Agrárias e Biológicas, 4(2), 12-20.

Lazzareti, E., \& Bettiol, W. (1997). Tratamento de sementes de arroz, trigo, feijão e soja com um produto formulado a base de células e de metabólitos de Bacillus subtilis. Scientia Agricola, 54(1-2), 89-96. doi:10.1590/S0103-90161997000100013
Lazzaretti, E. (2005). Influência de Bacillus subtilis na promoção de crescimento de plantas e nodulação de raízes de feijoeiro. Boletim de Pesquisa e Desenvolvimento No 28. Embrapa Ambiente, pp. 15.

Luz, W. C. (1996). Rizobactérias promotoras de crescimento de plantas e de bioproteção. Revisão Anual de Patologia de Plantas, 4, 1-49.

Machado, J. C. (2000). Tratamento de sementes no controle de doenças. Lavras: LAPS/ UFLA/ FAEPE, pp. 138.

Machado, D.F. M., Tavares, A. P., Lopes, S. J., \& Silva, A. C. F. (2015). Trichoderma spp. na emergência e crescimento de mudas de cambará (Gochnatia polymorpha (Less.) Cabrera). Revista Árvore, 39(1). doi:10.1590/0100-67622015000100016

Marques, E., Santos, D. B., Silva, J. B. T., Martins, I., \& Mello, S. C. M. (2014). Avaliação do tratamento biológico com isolados de Trichoderma spp. na germinabilidade de sementes de feijão. Cadernos de Agroecologia, 9(3).

Nakagawa, J. Testes de vigor baseados na avaliação das plântulas. In: Krzyzanoski, F.C., Vieira, R.D. \& França Neto, J.B. (1999). (Ed.). Vigor em sementes: conceitos e testes. Londrina: Abrates, pp. 2.1-2.24.

Oliveira, G. R. F., Silva, M. S., Marciano, T. Y. F., Proença, S. L., \& Sá, M. E. (2016). Crescimento inicial do feijoeiro em função do vigor de sementes e inoculação com Bacillus subtilis. Brazilian Journal of Biosystems $\quad$ Engineering, $\quad 10(4), \quad 439-448$. doi:10.18011/bioeng2016v10n4p439-448

Oliveira, L. M. de., Schuch, L. O. B., Bruno, R., \& Peske, S. T. (2015). Qualidade de sementes de feijão-caupi tratadas com produtos químicos e armazenadas em condições controladas e não controladas de temperatura e umidade. Semina: Ciências Agrárias, 36(5). doi:10.5433/16790359.2015v36n3p1263

Ramamoorthy, V., Viswanathan, R., Raguchander, T., Prakasam, V., \& Samiappan, R. (2015). Induction of systemic resistance by plant growth promoting rhizobacteria in crop plants against pests and diseases. Crop Protection, 20(1), 1-11. doi:10.1016/S0261-2194(00)00056-9

Reis, G. G., \& Muller, M. W. (1978). Análise de crescimento de plantas mensuração do crescimento. Belém, pp. 35.

Rocha, W. S., Sakai, T. R., Souza, D. L. A., Junior, A. F. C., \& Santos, M. M. (2017). Efeito da microbiolização na germinação e crescimento inicial de feijão caupi no Estado do Tocantins. Tecnologia \& Ciência Agropecuária, 11(6), 41-47.

Silva, M. C. A., Santos, M. M., \& Santos, M. G. (2018). Uso de bioestimulante em caupi via semente e foliar em diferentes fases vegetativas. Tecnologia \& Ciência Agropecuária, 12(1), 37-42.

Tarnawski, S., Hamelin, J., Jossi, M., Aragno, M., \& Fromin, N. (2006). Phenotypic structure of Pseudomonas populations is altered under elevated pCO2 in the rhizosphere of perennial grasses. Soil Biology \& Biochemistry, 38(6), 1193-1201. doi:10.1016/j.soilbio.2005.10.003

Yoshikawa, M., Hirai, N., Wakabayashi, K., Sugizaki, H., \& Iwamura, H. (1993). Succinic and lactic acids as plant growth promoting compounds produced by rhizosphere Pseudomonas putida. Canadian Journal of Microbiology, 39, 1150-1154. doi:10.1139/m93-173 